\title{
Die sexuelle Befreiung und das Problem mit der Lust. Ein Dialog
}

\author{
Tamara Lewin (Zürich) und Fabian Ludwig (Luzern)
}

Zusammenfassung: Der Begriff der «sexuellen Befreiung» ist an die 68er-Bewegung gebunden und findet heute nur noch selten Verwendung. "Frei» gelebte sexuelle Lust gilt nicht mehr als ein Mittel zur Befreiung aus den bürgerlichen Fesseln. Im Gegenteil gilt sie oftmals sogar als Herrschaftsinstrument, besonders wenn es um die Lust der Männer geht. Und wenn im Rahmen von \#metoo von "Freiheit» die Rede ist, dann geht es meist um Consent: Um die Freiheit, zu sexuellen Handlungen "Ja» oder "Nein» zu sagen. Geht es bei \#metoo um sexuelle Befreiung? Ist die Art, wie wir begehren, an unser Geschlecht gebunden? Und wenn ja, warum? Was bedeutet Consent im Bett und auf der Couch? Diese Fragen wollten die Autorin und der Autor im Dezember 2018 im Rahmen eines Vortrags am Psychoanalytischen Seminar Luzern diskutieren. Dieser ist hier in gekürzter Fassung wiedergegeben.

Schlüsselwörter: Sexuelle Befreiung, Consent, \#metoo

T: Stellen Sie sich vor, Sie hätten nächste Woche Abstimmungsunterlagen in Ihrem Briefkasten und müssten über die Einführung eines Gesetzes abstimmen, das die Einvernehmlichkeit sexueller Interaktion regelt. Kann man Sexualität gesetzlich regeln? Was würde es bedeuten? Was würde es bewirken? Wie Sie wissen, ist dieses Gedankenexperiment keineswegs aus der Luft gegriffen. In den letzten Wochen und Monaten haben wir beide uns intensiv mit der Frage beschäftigt, was es mit dem «affirmative consent» und der Sexualität, dem Geschlechterverhältnis, mit \#metoo \& Co. auf sich hat. Und wie sich die kollektive Psychodynamik dabei verstehen lässt. Von dieser Auseinandersetzung wollen wir heute Abend erzählen und versuchen, den Weg unserer Diskussion mit ihren Abzweigungen, Kreuzungen, Knotenpunkten usw. nachzuzeichnen.

F: Erwarten wird Sie also keine fertige Abhandlung, keine runde Sache. Erwarten wird Sie unser Stand der Verwirrung in diesen Fragen, ein Abbild unserer Diskussion, die sich immer wieder im Kreis dreht und in Widersprüche verstrickt. 
Ausgangspunkt war ein Gesetz mit folgendem Inhalt, welches im Mai dieses Jahres in Schweden verabschiedet wurde und viele Diskussionen ausgelöst hat.

Wenn eine Person sexuelle Aktivitäten mit einer anderen Person aufnehmen will, und diese andere Person inaktiv bleibt oder mehrdeutige Signale aussendet, wird er oder sie herausfinden müssen, ob die andere Person zu diesen sexuellen Aktivitäten gewillt ist. (BBC, 2018, Übers. d.Aut.)

Zunächst jedoch eins nach dem anderen. Ich weiss nicht, wie es Ihnen ergangen ist, als Sie den Vortragstitel gelesen haben. «Sexuelle Befreiung»: Das wirkt doch irgendwie aus der Zeit gefallen. Spricht heute noch jemand von der «sexuellen Befreiung»? Woran denkst Du bei dem Begriff, Tamara?

T: Gute Frage. Als erstes natürlich an Schlaghosen und wallendes Haar. Aber das meinst Du vermutlich nicht. Sexuelle Befreiung bedeutet für mich irgendwie so etwas wie «mein Körper gehört mir» und «unsere Lust gehört uns», also eine Auflehnung gegen einen Machtanspruch, der von aussen kommt.

F: Bürgerliche Repression, die Triebunterdrückung als Grundlage der Kultur. Wir sind also mitten in der 68er-Bewegung, für die der PsychoanalytikerWilhelm Reich ein wichtiger Bezugspunkt war. Der kulturelle Zwang zum Triebverzicht galt als das ideologische Instrument der bürgerlich-faschistoiden Gesellschaft. Und frei gelebte Sexualität entsprechend als das Instrument zur Befreiung von bürgerlichen Fesseln. Die Sexualität sollte es also richten. Bei Reich klingt das so:

Die sexuelle Unterdrückung ist ein reaktionärer Faktor von grossem Gewicht, denn: [...] sie stützt die Familien- und Eheordnung, welche zu ihrem Bestande Verkümmerung der Sexualität erfordert; sie macht die Kinder und Jugendlichen den Eltern und auf diese Weise später die Erwachsenen der staatlichen Autorität und dem Kapital hörig, indem sie in den Unterdrückten autoritäre Ängstlichkeit hervorruft; sie lähmt die Kritik der Unterdrückten, denn die sexuellen Schwierigkeiten verbrauchen viel Energie, die sonst zur kritischen Verstandesarbeit verwendet würde; sie lähmt, indem sie die Menschen scheu und unentschlossen macht, die revolutionären Kräfte im Individuum. (Reich 1934/35, nach Erdheim 1988, S. 219) 
T: Das ist ja eigentlich schon interessant: Es klingt, als gäbe es die Sexualität, die wahre oder eigentliche sozusagen, die es nur freizulassen gilt, und dann wird alles gut. Irgendwie scheint mir das alles etwas komplizierter zu sein mit dem Begehren und so.

F: Der damalige Zeitgeist scheint mit einer Art Glauben verbunden zu sein, einem Glauben an die wahre, natürliche Menschlichkeit, und dann eben auch an die wahre, natürliche Sexualität. Und dass dann eben die Kultur diese wahre Natur unterdrückt und verbogen habe, und dass wir das bloss abzuwerfen brauchen, und dann ist der wahre Mensch wieder da. Und ein Weg, um diese kulturelle Deformation wieder abzuwerfen, war dann die frei gelebte Sexualität, die uns dann ja auch befreien sollte von all den anderen Kulturbürden. Kultur war Unterdrückung der Lust, und Lust war frei. Das ist sicherlich ein Glaube, der heute nicht mehr sehr verbreitet ist.

T: Schon in den feministischen Bewegungen der 70er Jahre wurde festgestellt, dass die sogenannt «frei gelebte Sexualität» oftmals gerade zur Reproduktion von patriarchalen Mustern führte. Dass die Lust und die Sexualität ganz grundsätzlich von den gesellschaftlichen Strukturen geformt und geprägt werden. In der WOZ erschien vor ein paar Wochen ein Artikel von der Philosophin Amia Srinivasan (Srinivasan 2018), welcher diese feministischen Bewegungen nachzeichnete. Die Feministin Catharine MacKinnon meinte Ende der 70er-Jahre, Sex sei im Patriarchat immer gewalttätig. Die ganze Struktur des Sex sei durch die patriarchalische Herrschaft bestimmt. Und dass es Frauen gebe, die unter diesen Bedingungen Lust an Sex erfahren können, sei ein Zeichen dafür, wie schlimm die Dinge stünden. Demgegenüber entwickelten sich in den 80er- und 90er-Jahren feministische Strömungen, die dieser Haltung eine «sexpositive» Perspektive entgegenhielten. Sie setzten sich für das Recht der Frauen auf Sex ohne Schuldgefühle ein. Wenn eine Frau Lust darauf hat, von einem Mann beim Sex dominiert zu werden, dann soll sie das auch wollen und erleben dürfen.

F: Die Frauen sollten jetzt also beim Wort genommen werden, ihr Begehren sollte so akzeptiert werden, wie es nun einmal sei. Der sexpositive Feminismus hat sich heute durchgesetzt. Das Begehren wird so hingenommen, wie es halt ist, und die einzige moralische Grenze ist die beidseitige Zustimmung - der Consent also. 
T: Im erwähnten WOZ-Artikel wird hier eine Nähe zum kapitalistischen Austausch postuliert: Der kapitalistische Liberalismus wie auch der sexpositive Feminismus hinterfragen nur ungern, wie wir zu unseren Wünschen kommen. So wie sich der Durchschnittskonsument nicht gross fragen soll, wie denn nun sein dringender Wunsch nach einem MacBook zustande gekommen ist, so soll sich auch die Frau nicht fragen müssen, weshalb sie denn nun einen reichen, dominanten Mann begehrt. Hauptsache, alle Beteiligten geben ihre Zustimmung.

F: Und hier wird das Dilemma spürbar. Auf der einen Seite haben wir den sogenannten «Anti-Porno-Feminismus», und der Porno ist ja vielleicht gleich ein anschauliches Beispiel: Wenn eine Frau sagt, sie habe Lust darauf, in einem Porno mitzuspielen, dann würde eine solche Haltung bedeuten, besser zu wissen, was diese Frau eigentlich will. Man würde aus dieser Haltung heraus beispielsweise sagen, diese Frau täusche sich selbst mit ihren angeblichen Wünschen, und eigentlich würde sie etwas ganz Anderes wollen, hätten die patriarchalen Verhältnisse sie nicht schon derart gefügig gemacht.

Aus einer «sexpositiven» Haltung könnte man dagegenhalten, ein so verstandener Feminismus wirke gerade kontraproduktiv: Er führe zu einer Konstellation, in der diese Frau von neuem entmündigt wird, indem ihr vorgeschrieben werde, was sie zu wünschen habe und was nicht.

T: Das Problem einer derart unkritischen Einstellung zu unseren Wünschen ist dann aber wiederum, dass alles beim Alten bleibt: Weiter werden massenhaft Pornos produziert, welche Frauen zeigen, die sich dem Anschein nach willig unterwerfen. Weiter konsumieren Jugendliche diese Art von Pornos und werden in ihren sexuellen Wünschen dadurch geprägt, usw.

Das Schöne an dem WOZ-Artikel ist, dass er nicht in dieser Polarisierung bleibt, sondern die Unauflösbarkeit des Konflikts anerkennt. Das Verfolgen und Geniessen der eigenen Wünsche soll nicht daran hindern, diese zu befragen.

Ja-Wie entstehen unsere Wünsche? Da kommt die Psychoanalyse ins Spiel, und hier wäre wohl der Punkt, wo man in einem ordentlichen Referat mal mit einem Wörterbuch-Eintrag beginnt. Ich versuch es mal mit Laplanche \& Pontalis:

Jede Konzeption vom Menschen hat Begriffe, die zu fundamental sind, als dass man sie genau bestimmen könnte. Dies trifft in der Freudschen Doktrin zweifellos für den Wunsch zu, (Laplanche \& Pontalis 1972, S. 635) 
Gar nicht so einfach mit Freud also. Lassen Sie mich einen Zeitsprung machen und ein wenig ausholen, wenn ich ein Stück weit der Argumentationslinie der feministischen Psychoanalytikerin Jessica Benjamin folge, wie sie in «Die Fesseln der Liebe» (Benjamin 1990) meines Erachtens sehr einleuchtend dargestellt ist.

Benjamin argumentiert im Grunde so:

, Das Problem des weiblichen Begehrens ist, dass Frauen in unserer Kultur nicht eigentlich ein eigenes Begehren haben, sondern ihnen nur übrigbleibt, das Begehren des Mannes zu begehren. Sie postuliert also ein männliches Monopol des Begehrens, symbolisch im Phallus ausgedrückt. , Diese Konstellation sieht sie nicht als Folge des anatomischen Geschlechtsunterschieds, sondern als Kulturprodukt. Primäres Problem dieser Kultur ist, vereinfacht gesagt, wie sich die Beziehung des Mädchens zum Vater gestaltet, nämlich im Kontext der bestehenden Geschlechterpolarität und deren Konsequenzen für die Erziehung.

, Mit der kulturell produzierten Geschlechterpolarität geht einher, dass «weiblich-sein» grob und plakativ gesagt nur zwei Formen kennt. Entweder - und in erster Linie - ist die Frau Mutter. Und dieses Muttersein ist kulturell spezifisch aufgeladen: «Die Mutter ist eine vollständig entsexualisierte Figur», schreibt sie, und weiter: «Sie mag Kontrolle und Macht über andere haben, nicht aber über ihr eigenes Schicksal» (Benjamin 1990, S. 88). Das Gegenbild zur mächtigen und heiligen Mutter wäre dann etwa die «femme fatale». Doch auch diese drückt kein eigenes Begehren aus, sondern zelebriert vielmehr die Lust, begehrt zu werden.

, Wie erklärt Benjamin jetzt das Problem des mangelnden weiblichen Begehrens? Ich zitiere ihre These: «Was Freud als Penisneid bezeichnete, nämlich die männliche Orientierung des kleinen Mädchens, reflektiert in Wirklichkeit den Wunsch von Kindern beiderlei Geschlechts, sich mit dem Vater zu identifizieren, der als Repräsentant der Aussenwelt erlebt wird» (Benjamin 1990, S. 99).

, Der entscheidende Punkt liegt also in den Vorgängen der Identifikation bzw. im Fehlen pluraler Identifikationsangebote. Unsere sozialen Strukturen sind weiterhin stark geprägt durch die Kopplung «Mann - aktiv (im) Aussen» und «Frau - passiv - (im) Innen». Im Zuge der Individuation, also der Loslösung von der Familie stellt sich so auch heute noch für Mädchen im Unterschied zu Jungen die Frage: Wie kann ich dem Vater 
ähnlich sein, also in die Welt hinausgehen und etwas wollen, und trotzdem eine Frau sein?

F: Also, mal ganz vereinfacht: «Die selbstlose Mutter ist zu Hause am Herd, und der Vater ist in der aufregenden Welt unterwegs.» Als Vorbild für die Ablösung von der Familie kann dann einzig derVater dienen. Dem Mädchen fehlen Modelle für selbstbestimmtes weibliches Begehren. Die von Freud beschriebenen psychischen Strukturen - wie eben beispielsweise der «Penisneid» - sind für Benjamin keine anthropologischen Grundkonstanten, sondern letztlich der psychische Abdruck von gesellschaftlichen Strukturen. Eine solche Sichtweise legt den Schwerpunkt auf gesellschaftlicheVeränderungen, die wichtig sind, um selbstbestimmtes weibliches Begehren zu erleichtern: Väter in der Familie und Mütter in der Aussenwelt, mehr weibliche Akteure in Büchern und Filmen, usw.

T: Macht es dann noch Sinn, über Fragen des Consent zu verhandeln?

F: Ich glaube, ja. Natürlich ist es für uns alle prägend, wie wir aufwachsen, welche Rollenbilder uns vorgelebt werden. In der Adoleszenz tun sich dabei nochmals viele Möglichkeiten auf. Ich glaube, die sexuellen Erlebnisse - oder Nicht-Erlebnisse in der Jugendzeit sind mindestens so bedeutend für die Entwicklung der eigenen Sexualität wie die Kindheit. Es ist sicher eine schwierige Zeit für den Consent, ist man in dieser Zeit doch oft sehr damit beschäftigt, ob man dazugehört oder nicht, was man gerne wäre und was sicher nicht. Oft ist es nicht die Zeit für eine authentische Befragung der eigenen Wünsche und Verletzlichkeiten. Man will «es» wissen, und die Frage, ob einem das denn nun eigentlich gefällt oder nicht, die ist oft hintangestellt. Und doch, und auch gerade darum, ist es nach meinem Empfinden wichtig, welche Haltung die Gesellschaft zu Fragen des Consent einnimmt, gerade in ihrer Rechtsprechung. Ich glaube aber nicht, dass sich diese Fragen auf einer abstrakten Ebene diskutieren lassen.

T: Fabian hat mir neulich das Buch von Annie Ernaux, «Erinnerung eines Mädchens», zum Lesen gegeben (Ernaux 2018). Die Autorin beschreibt darin mit 50 Jahren Verschiebung, also in der Nachträglichkeit par excellence, ihre ersten sexuellen Erfahrungen als 18-jährige in einer Ferienkolonie, in der sie als Kinderbetreuerin dabei war. Die Komplexität des Themas Consent zeigt sich hier sehr schön, und ich möchte gerne einige der Schlüsselszenen vorlesen. 
In der Folgenden Stelle beschreibt sie erstmals den Beginn der für sie nachhaltig einschneidenden Interaktion mit $\mathrm{H}$, einem der Chefbetreuer. Sie schreibt von ihrem 18-jährigen Ich in der dritten Person.

Seit drei Tagen ist sie in der Kolonie. Es ist Samstagabend. Die Kinder liegen in den Schlafsälen in ihren Betten. Ich sehe sie, wie später noch Dutzende Male, mit ihrer Zimmergenossin die Treppen hinunterlaufen, in Jeans, einem ärmellosen Ringelpulli, weissen Riemchensandalen. Sie hat die Brille abgesetzt und den Dutt gelöst, die langen Haare wippen in ihrem Rücken. Sie ist in einer extremen Aufregung, es ist ihre erste Party.

Ich weiss nicht mehr, ob schon Musik lief, als die beiden in dem Keller ankamen, der in einem Nebengebäude lag, vielleicht unter der Krankenstation oder einem anderen Raum. Und auch nicht mehr, ob er schon da war, ob er einer von denen war, die sich um den Plattenspieler drängten und die Musik auswählten. Sicher ist nur, dass er der Erste war, der sie zum Tanzen aufgefordert hat. Ein Rock'n'Roll.

Es ist ihr peinlich, wie schlecht sie tanzt (womöglich hat sie es ihm gesagt, um sich zu entschuldigen). Sie wirbelt mit grossen Schritten um ihn herum, geführt von seinem Griff, ihre Sandalen machen klack klack auf dem Betonboden. Sie ist aufgewühlt, weil er sie unverwandt anstarrt, während er sie eine Drehung nach der anderen machen lässt. Noch nie hat jemand sie mit so schweren Augen angeschaut. Er, das ist $H$, der Chefbetreuer. Er ist gross, blond, breitschultrig, mit kleinem Bauch. Sie fragte sich nicht, ob er ihr gefällt, ob sie ihn schön findet. Er wirkt kaum älter als die anderen Betreuer, aber für sie ist er kein Junge mehr, sondern ein richtiger Mann, wegen seiner Stellung, nicht so sehr wegen seines Alters. Wie sein weibliches Pendant, die Chefbetreuerin L, steht er für sie auf der Seite derjenigen, die das Sagen haben. Mittags hat sie am selben Tisch wie er gegessen, eingeschüchtert, sehr beschämt, weil sie nicht wusste, wie man den Pfirsich, den es zum Nachtisch gab, richtig isst. Nicht eine Sekunde lang hätte sie gedacht, dass er sich für sie interessieren könnte, sie ist perplex. 
Beim Tanzen weicht er zur Wand zurück und hört dabei nicht auf, sie anzustarren. Das Licht geht aus. Er zieht sie abrupt an seine Brust und presst seinen Mund auf ihren. In der Dunkelheit wird laut protestiert, jemand macht das Licht wieder an. Ihr wird klar, dass er es war, der den Schalter betätigt hat. Sie empfindet eine wohlige Angst und kann ihm nicht in die Augen sehen. Sie weiss nicht, wie ihr geschieht. Er flüstert, gehen wir raus? Sie sagt ja, vor den anderen können sie sich nicht küssen. Sie sind draussen, laufen eng umschlungen an den Mauern des Sanatoriums entlang. Es ist kalt. In der Nähe des Speisesaals, vor dem dunklen Park, drückt er sie gegen die Mauer, reibt sich an ihr, sie spürt seinen Penis durch ihre Jeans an ihrem Bauch. Es geht ihr zu schnell, sie ist nicht bereit für diese Geschwindigkeit, diese Gier. Sie spürt nichts. Sie lässt sich von seinem Begehren unterwerfen, dem unbeherrschten, wilden Begehren eines Mannes, das nichts zu tun hat mit dem langsamen, behutsamen Flirt, den sie im Frühling erlebt hat. Sie fragt nicht, wohin sie gehen. Wann wird ihr klar, dass er sie zu den Zimmern führt, vielleicht hat er es ihr gesagt?

Sie sind in ihrem Zimmer, im Dunkeln. Sie sieht nicht, was er tut. In diesem Moment glaubt sie immer noch, dass sie sich auf dem Bett weiterküssen und durch die Kleidung hindurch streicheln werden. Er sagt: "Zieh dich aus.» Seit er sie zum Tanzen aufgefordert hat, hat sie alles getan, was er von ihr verlangt hat. Zwischen dem, was ihr passiert, und dem, was sie tut, gibt es keinen Unterschied. Sie legt sich neben ihn auf das schmale Bett, nackt. Sie hat keine Zeit, sich an seine absolute Nacktheit zu gewöhnen, an seinen nackten Männerkörper, sofort spürt sie die Grösse und Härte seines Glieds, das er ihr zwischen die Schenkel schiebt. Er versucht, in sie einzudringen. Es tut ihr weh. Sie sagt, dass sie noch Jungfrau ist, als Verteidigung oder Erklärung. Sie schreit. Er beschwert sich: «Mir wäre es lieber, du würdest vor Lust schreien!» Sie wäre gern woanders, aber sie bleibt. Ihr ist kalt. Sie könnte aufstehen, das Licht anmachen und ihm sagen, er solle sich anziehen und verschwinden. Oder sich selbst anziehen, ihn dort zurücklassen und wieder auf die Party gehen. Sie hätte das tun können. Ich weiss, dass sie nicht auf die Idee gekommen ist. Als wäre es zu spät, es sich jetzt 
noch anders zu überlegen, als müssten die Dinge ihren Lauf nehmen. Als hätte sie nicht das Recht, diesen Mann in dem Zustand zurückzulassen, den sie in ihm ausgelöst hat. Mit dieser rasenden Lust. Für sie ist es unvorstellbar, dass er sie unter all den anderen ausgesucht hat-auserwählt.

Die Fortsetzung läuft ab wie ein Pornofilm, in dem der Mann den Takt vorgibt und seine Partnerin nicht weiss, was sie tun soll, weil sie keine Ahnung hat, was als Nächstes kommt. Er allein ist Herr der Situation. Er ist ihr immer einen Schritt voraus. Er schiebt sie nach unten, in Richtung seines Bauchs, steckt ihr seinen Schwanz in den Mund. Sofort schwappt ihr ein fetter Schwall Sperma entgegen, spritzt ihr bis in die Nasenlöcher. Es sind keine fünf Minuten vergangen, seit sie das Zimmer betreten haben.

In meiner Erinnerung kann ich kein Gefühl finden, geschweige denn einen Gedanken. Das Mädchen auf dem Bett nimmt an dem Geschehen teil, mit ihr passiert etwas, was sie eine Stunde zuvor nicht für möglich gehalten hätte, mehr nicht. (Ernaux 2018, S. 43-45)

F: Hier ist etwas passiert, etwas, was das Leben und die Sexualität der Autorin ein Leben lang prägen sollte, wie sie selbst schreibt. Was aber ist denn passiert? Eine Traumatisierung, wird man heute vermutlich denken, eine Vergewaltigung. Bei Ernaux selbst findet sich auch 50 Jahre später keine klare Einordnung. Sie schämt sich für die Szene, wie sie schreibt, kann lange nicht darüber sprechen und nur mit viel Mühe darüber schreiben. Zugleich hat die Nacht bei ihr etwas in Gang gesetzt. Was in dieser Nacht passiert ist, will sie mit H wiederholen, sucht dann sexuelle Kontakte und Anzüglichkeiten auch mit anderen Jungen und Mädchen, und gilt zuletzt in der Gruppe der Betreuer und Betreuerinnen als das «leichte Mädchen».

T: Heute würde man das wohl Slutshaming nennen. Und damit ist auch gesagt, dass es sich dabei nicht um ein Ding der Vergangenheit handelt. Die Ereignisse im Buch fanden im Jahr 1958 statt. Aber auch heute noch werden Frauen, die ihr Begehren zeigen und äussern, als «Schlampen» beschämt. Was bei Männern so hingenommen wird - «Jungs sind halt Jungs» - ist bei Frauen in weiten Kreisen der Gesellschaft nach wie vor eine Unart. 
F: Erzeugt das Begehren der Frauen denn so viel Angst, dass man es derart entwerten muss?

T: Müssen wir uns nicht vielmehr fragen, ob wir es hier eigentlich überhaupt wirklich mit Begehren zu tun haben? Ich finde, im Buch wird hier eben gerade so deutlich, wie schwierig das mit dem Begehren ist. Wie diffus die Grenze zwischen Zwang undWollen. Etwas ist mit Annie Ernaux passiert, was sie nicht für möglich gehalten hätte - «mehr nicht». Was fehlt, ist eine Bezeichnung für das, was passiert ist, eine Einordnung: «In meiner Erinnerung kann ich kein Gefühl finden, geschweige denn einen Gedanken». Könnte man nicht genau hier sagen, dass dieser jungen Frau hier nichts anderes übrigbleibt, als das, was ihr geschieht, zu einer Art Begehren zu machen? Aus der Not eine Tugend? Oder macht man sie so erst zum Opfer, das sie gar nicht ist? Sie schreibt an einer Stelle auch, dass es ihr unmöglich war, zum Orgasmus zu kommen und dass es nicht denkbar war, ihren Wunsch nach oraler Befriedigung zu äussern. Aber auch wenn die Szenerie zunächst eindeutig durch sein Begehren dominiert ist, könnte man natürlich dennoch fantasieren, dass es für ihn ebenso wenig die Möglichkeit gab, sich in andererWeise sexuell zu erleben und zu zeigen.

F: Das stimmt, man kann sich ja schon fragen, was das denn für ein Begehren ist, das ihn antreibt. Es macht ja den Anschein, dass er sich seiner Männlichkeit versichern muss, die er als Macht und Potenz versteht: «Vor Lust schreien» soll seine Eroberung.

T: Er will erobern, sie will erobert werden.

F: Oft wird dieses Liebesspiel vom «erobern» und «erobert werden» ja anthropologisch begründet, mit biologischen «Wahrheiten» über das «Mann-sein» und das «Frau-sein». Solche «Wahrheiten» finden sich auch in der Psychoanalyse, und Freud hat sie in Totem und Tabu auch in die Urgeschichte der Menschheit verlegt. In seiner Einleitung zu Totem und Tabu schlug Mario Erdheim eine Lektüre dieser Urgeschichte vor, die mich in diesem Zusammenhang interessiert, weil sie der Urgeschichte eine Geschichte der Gewalt entgegenstellt, und damit vielleicht auch das etablierte Liebesspiel zwischen Männern und Frauen auf neue Weise lesbar macht: 
der Vatermord war nicht die Tat, die ungeschehen gemacht werden sollte, sondern er ist der Mythos, der die bestehenden und zukünftigen Ungleichheiten rechtfertigt. Was Freud beschreibt, sind dann zum einen Prozesse, die die Unbewußtmachung einschneidender und gewaltsamer Umstürze und Machtverlagerungen erwirken, zum anderen erklärt er, wie es möglich war, hierarchische Ordnungen aufzubauen, die eine Mehrheit zugunsten einer Minderheit entmachteten.

Der erste große Entmachtungsschub fand wahrscheinlich im Verhältnis zwischen den Geschlechtern statt und zerbrach die Gegenseitigkeit zwischen Männern und Frauen. Die Männer konnten sich allmählich und später immer schneller eine privilegierte Stellung aufbauen. Ein späterer Entmachtungsschub betraf dann ganze Bevölkerungsteile, was zur Entstehung sozialer Schichten und Klassen führte. Zweifellos gingen diese Entmachtungsprozesse gewaltsam vor sich. Und Hand in Hand mit der Gewalt ging deren Unbewußtmachung. (Erdheim 1991, S. 36-37)

Dem Liebesspiel - zwischen dem Mann, der erobern will, und der Frau, die erobert werden will - kommt vielleicht eine ähnliche Rolle zu: Die Rolle der Rechtfertigung von bestehenden und zukünftigen Ungleichheiten. Indem die Ungleichheit ins erotische Spiel übertragen wird, erscheint sie als etwas «Naturgegebenes», als etwas «nur allzu Menschliches». Die Geschichte der Gewalt wird damit vergessen gemacht; an ihre Stelle tritt die «ewige Geschichte» von Männlein und Weiblein, als eines dieser Dinge, die sich scheinbar nie ändern.

T: Die Art und Weise, wie Frauen und Männer zu Lust kommen, ihre konfliktbehafteten sexuellen Wünsche, all dies erweist sich in einer solchen Sichtweise als Folge und als Träger von gesellschaftlichen Interessen, von Machtansprüchen.

Jetzt frage ich mich aber:Wie würden denn die sexuellenWünsche und Begegnungen aussehen in einer Gesellschaft, in der solche Machtansprüche beseitigt wären? Landen wir dann nicht wieder bei der idealen, «wahren» Sexualität, beim «wahren Mensch-sein»? Wäre die Sexualität für solche «wahre Menschen» denn eine konfliktfreie Sache? Gerade dies wird den Befürwortern von Consent-Gesetzen von 
polemischen Kritikern wie Slavoj Žižek ja immer vorgeworfen: Dass sie von einer bereinigten, konfliktfreien Sexualität träumen, welche aber letztlich nicht mehr viel mit Sexualität zu tun hätte.

F: Ich glaube, es hilft, wenn man das Wünschen als etwas Zusammengesetztes versteht. Das kann man auf unterschiedliche Weise tun. Man kann es mit Freud halten: Auf der einen Seite stehen dann die autoerotischen Partialtriebe, welche anarchistisch organisiert sind und «jeder für sich, die Lustbefriedigung am eigenen Leibe» suchen: Orale und anale Triebe, Schautrieb, Zeigelust, Bemächtigungstrieb, usw. Auf der anderen Seite steht bei Freud die genitale Sexualität, die «Erwachsenensexualität» sozusagen, in welcher diese anarchistischen Triebe gebündelt und kanalisiert werden, auf ein vermeintlich ganzes Ich und ein ganzes Gegenüber - womit sie dann auch «gesellschaftsfähig» werden.

Slavoj Žižek hat das Zusammenspiel dieser beiden Ebenen im Sex in einem älteren Buch einmal auf witzige Weise dargelegt:

«Reifer» Sex zwischen den sprichwörtlichen mündigen Erwachsenen [...] ist per definitionem entsexualisiert und verwandelt sich in mechanisches Kopulieren. (Žižek 2010, S. 396).

Erregend wird dieses Kopulieren erst mit der Zergliederung der sexuellen Begegnung durch die Partialtriebe - in diese ganz bestimmte Berührung, jenen ganz bestimmten Blick, eine bestimmte Art zu Küssen usw. Partialtriebe wie der Schautrieb und die kindliche Sexualneugier leisten für Žižek einen unverzichtbaren Beitrag zur Erwachsenen-Sexualität, sodass ...

...sogar zwei mündige Erwachsene, die hinter geschlossenen Schlafzimmertüren «normalen und gesunden» Sex miteinander haben, niemals ganz unter sich sind: Es gibt immer einen "phantasmatisierten» Blick des Kindes, der sie beobachtet, ein - gewöhnlich «internalisierter» - Blick, aufgrund dessen ihr Tun für sie selbst letzten Endes ein undurchdringliches bleibt. (Žižek 2010, S. 396)

T: Beim Sex ist man für Žižek also immer zugleich auch ein Kind, welches einem erregt durch das Schlüsselloch zuschaut, ohne zu verstehen, was hier vor sich geht. Die Partialtriebe liefern also die phantasmatischen Stützen, welche den «Erwachsenensex» überhaupt erst erregend machen. 
F: Fritz Morgenthaler hat, so wie ich ihn verstehe, den Bestandteilen des Wünschens andere Namen gegeben. Er unterscheidet «das Sexuelle» von «der Sexualität». In seinem Aufsatz «Sexualität und Psychoanalyse» schreibt er:

Der Primärprozess ist in erster Linie durch die Ungerichtetheit der dem Es zugehörigen Triebregungen bestimmt, während der Sekundärprozess alles Triebhafte in Bahnen lenkt, organisiert und nach anzustrebenden Zielsetzungen ausrichtet. Sprechen wir von Sexualität im Gegensatz zum Sexuellen, handelt es sich um das, was der Sekundärprozess aus den Triebregungen im Es gemacht hat. (Morgenthaler 1994, S. 146)

Die Sexualität ist für Morgenthaler die Form, in der das Triebhafte gesellschaftsfähig wird, also kulturell vermittelt und vermittelbar, und damit in gewisser Weise auch gebändigt und kanalisiert. Die Sexualität meint bei Morgenthaler also dieWeise, in der wir die anarchistische Triebhaftigkeit symbolisieren und in geordnete Bahnen zu lenken versuchen. Dies mag aber nie so ganz gelingen.

Es besteht eine Disharmonie zwischen den primärprozesshaften Triebregungen und der sexuellen Diktatur, die den Unterschied zwischen dem, was wir das Sexuelle, und dem, was wir Sexualität nennen, ausmacht. [...] Das Potential der Triebe, die sich der sekundärprozesshaften Kanalisierung entziehen, bleibt dauernd freischwebend disponibel, um sich als emotionale Bewegung allen möglichen Ichfunktionen momentan und reversibel beizumischen. (Morgenthaler 1994, S. 46)

Anders als bei Wilhelm Reich verstehe ich diese «Diktatur» der Sexualität bei Morgenthaler nicht als eine, die wir abwerfen können. Zwar kann sich die Sexualität kulturell verändern, aber die Grundstruktur der Disharmonie im Wünschen bleibt bestehen, weil der Wunsch erst im Übergang in die Sprache, in die Vergesellschaftung entsteht. Ob man von den anarchistischen Partialtrieben oder vom Sexuellen bei Morgenthaler ausgeht: Bei der Übersetzung des Triebhaften in eine gesellschaftlich vermittelbare Sexualität entsteht der Wunsch als disharmonische Konstruktion, die hinten und vorne nicht aufgeht.

Darin liegt, glaube ich, das Konflikthafte und Übergriffige, das an der Sexualität immer haften bleibt. Das Übergriffige im Sinne der Übertragung: Ein Übergriff 
vom Autoerotismus auf die «Erwachsenensexualität», von der Vergangenheit auf die Gegenwart, vom Unbewussten auf das Bewusste.

T: Das heisst also, es gilt zu unterscheiden zwischen (1.) dem, was in der Sexualität immer schon konflikthaft und durch einen Bruch gekennzeichnet ist, nämlich dass wir den Sprung vom rein Triebhaften in Sprache und Fantasie vollziehen müssen, und (2.) der konkreten Sprache, die kulturell kontingent bereitgestellt wird, also den Codes, die in unserem Fall durch Machtstrukturen entlang des Geschlechtsunterschieds geprägt sind.

F: Dazu finde ich auch eine Unterscheidung hilfreich, die Mario Erdheim im Aufsatz «Das Unbewusste in der Geschichte» getroffen hat. Erdheim ordnet dem Unbewussten zwei Funktionen zu:

\begin{abstract}
Die eine lässt sich mit dem Begriff des Es bezeichnen und umfasst die triebhaften Seiten des Unbewussten, während die andere Funktion durch die einfrierende, konservierende Kraft des Unbewussten zustande kommt. Das Unbewusste als Es treibt das Individuum immer wieder an, neue Objekte der Lust zu suchen und zu schaffen; das Unbewusste als Konservator nimmt hingegen diejenigen Elemente auf, die das Ich an der Realität scheitern liessen, und konserviert sie als unbewusst gewordener Wunsch. (Erdheim 1988, S. 169)
\end{abstract}

Vom Liebesspiel nach dem Muster der Eroberung könnte man sagen, dass es seine Kraft aus der konservierenden Funktion des Unbewussten bezieht, aus dem gesellschaftlichen Bruch zwischen den Geschlechtern, welcher immer wieder unbewusst gemacht und damit eingefroren werden muss.

T: Ich glaube, jetzt verstehe ich auch, was mich an den aktuellen Beiträgen von Slavoj Žižek stört. In seinem Artikel in der NZZ zum Beispiel, mit dem Titel «Das Leben ist nun einmal krass» (Žižek 2017), argumentiert er, Consent-Gesetze seien der neueste Auswuchs einer grassierenden «Political Correctness». Den Befürwortern schwebe eine Sexualität vor, die es gar nicht geben könne, und zwar eben eine bereinigte, eine konfliktfreie Sexualität. So verstanden wollten die Befürworter jegliche Sexualität auf die gegenseitige vertragliche Einigung reduzieren, und würden nicht merken, dass es gerade das Konflikthafte und das Übergriffige sei, was 
die Sexualität ausmache. Wenn Žižek argumentiert, mit der «politisch korrekten» Handhabung - man könnte auch sagen: Demokratisierung - der Sexualität gehe ihr eigentliches Wesen und somit ihr Reiz verloren, ist dies in zweierlei Hinsicht bemerkenswert: Erstens wäre zu begründen, weshalb Schranken des Lustgewinns nicht auch gelegentlich in Kauf zu nehmen sein sollten.

Die interessantere Frage aber ist, warum Kommunikation darüber, was in einer sexuellen Begegnung gewünscht ist und was nicht, automatisch den Spass verderben soll. Es lässt sich vermuten, dass dies nur mit der (nach wie vor sehr verbreiteten) Vorstellung festgeschriebener Positionen des Begehrens zu erklären ist. Žižek argumentiert hier mit der Errungenschaft, dass sich Frauen heutzutage endlich auch selbstbewusst, selbstgewählt, autonom-spielerisch sozusagen als Objekt anbieten und wieder entziehen können.

F: Zum Beispiel erwähnt er repressive Regierungen im arabischen Raum, welche Frauen das Spielen mit Bananen und anderen penisförmigen Gegenständen verbieten. Im Gegensatz dazu hätten die Frauen im Westen alle gesetzliche Freiheit, mit den Männern zu spielen und so die Machtverhältnisse zu unterwandern. Genau darin liege denn auch die Errungenschaft der sexuellen Befreiung der Frau.

T: Tatsächlich ist gegen Bananen ja nichts einzuwenden - sie als ultimatives Instrument der sexuellen Befreiung der Frau zu postulieren, scheint mir jedoch zu einfach. Denn zu bedenken ist doch, dass eine Wahl das Vorhandensein von Optionen bedingt. Hier ist der Punkt, an dem das eigentliche Problem oftmals übersprungen wird: Das Festhalten an einer zweigeschlechtlichen und heteronormativen Ordnung, in der die Geschlechtszugehörigkeit weiterhin mit einer klar zugeschriebenen Begehrensweise gekoppelt ist

Žižek nimmt also in seinen polemischen Beiträgen eine Gleichsetzung zwischen zwei verschiedenen Ebenen vor: Eine Gleichsetzung zwischen dem Übergriffigen, wie es der Sexualität in ihrer Abhängigkeit vom Sexuellen eigen ist, und dem systematisch Übergriffigen der patriarchal geprägten Sexualität.

F: Er vermischt also die zwei Funktionen des Unbewussten, so wie sie Mario Erdheim formuliert hat.

T: Dennoch bin ich immer noch skeptisch, wenn es um Consent-Gesetzgebung geht. Judith Butler fragt in einem Essay zu diesem Thema, im Anschluss an Foucault: 
If there is sexual freedom [...] it does not take the form of consent [...] Are there other languages for thinking about the sense of freedom that one might hope is captured by the language of consent? (Butler 2011)

Und:

So perhaps for all kinds of reasons it is time to rethink and displace the exclusive opposition between moral protectionists and libertarians. Perhaps that framework is no longer sufficient for us. And is the language of consent so corrupt and abused that it is no longer of use? I think in fact it circulates surreptitiously even in the arguments against consent. (Butler 2011, S 14)

In diesem Text bezieht sie sich auf die Consent-Debatte in den USA, insbesondere auf Consent-Gesetze an amerikanischen Universitäten, welche ja viel schärfer formuliert sind als etwa die neue Schwedische Gesetzgebung, und wo es dann teilweise wirklich um das Anfertigen von Sex-Verträgen geht.

Butler argumentiert weiter mit psychoanalytischen Konzepten gegen die libertäre Auffassung des Subjekts, die mit vertraglichen Regelungen impliziert wird. Das heisst, sie dekonstruiert das Bild von einem Menschen, dessen sexuellen Wünsche eindeutig sind, der also immer schon weiss, was er sich wünscht und was nicht, und auf dieser Grundlage frei entscheiden kann. Gerade in Hinblick auf verschärfte Consent-Gesetze an US-amerikanischen Universitäten warnt sie vor einem solchen Menschenbild, und sagt: So funktioniert das nicht mit unserem Wünschen. Zum Beispiel kann es sein, dass wir Entscheidungen treffen, die zu einem bestimmten Bild von uns selbst passen, und wir uns auf etwas einlassen, was wir nachträglich als traumatische Erfahrung erkennen müssen. Umgekehrt kann es natürlich auch sein, dass wir plötzlich merken, dass wir auf eine Weise Lust empfinden, die überhaupt nicht zu unserem Selbstbild passt, was ja auch eine traumatische Erfahrung sein kann. Was wir wünschen und was nicht, was uns den «Kick» gibt und was traumatisch wirkt, das lässt sich oft erst nachträglich sagen, und auch dann manchmal nicht so genau. Darum muss die Gesetzgebung nach Butler auch Raum lassen für sogenannte «schlechte Entscheidungen», für Entscheidungen, die zu traumatischen Erlebnissen führen, ohne dass daraus ein Rechtsanspruch resultiert 
It is important to distinguish between sex that did not turn out the way one imagined it would and sex that that was unilaterally imposed and clearly against one's will. (Butler 2011, S. 20)

F: Da frage ich mich aber: Kann man Consent-Gesetze nur dann befürworten, wenn man ein libertäres Menschenbild pflegt? Es stimmt ja schon: «If there is sexual freedom, it does not take the form of consent». Consent ist nicht die sexuelle Befreiung. Consent macht das Wünschen und den Sex nicht konfliktfrei. Und doch kann der Consent ein hilfreiches Instrument sein. In der Szene beispielsweise, die Du vorgelesen hast, da kam es mir vor, als würde sich Annie Ernaux im freien Fall befinden. Wie wäre die Szene abgelaufen, wäre damals ein Consent-Gesetz in Kraft gewesen, hätten das Mädchen und der Betreuer in der Schule davon gehört? Ich könnte mir vorstellen, dass eine solche Rechtsprechung doch eine Stütze sein kann, damit das Ganze nicht einfach abläuft, wie es nun einmal abläuft, damit man sich fragen kann, was man denn nun mit dieser Situation machen will und was nicht. Sicherlich ist es auch ohne Gesetz möglich, sich diese Fragen zu stellen, doch wird dies vielleicht leichter und selbstverständlicher, wenn es darüber eine verbindliche gesellschaftliche Vereinbarung gibt.

T: Am Anfang von ihrem Text macht Judith Butler einen interessanten Vergleich. Im Grunde, meint sie, ist es die klinisch arbeitende Psychoanalytikerin, die zum Thema Consent am meisten zu sagen hat. Diese hat bei ihrer Arbeit Einblick in die Situation, dass ein Analysand freiwillig zur Analyse kommt und sein Einverständnis mit seinem Erscheinen in jeder folgenden Sitzung auch erneuert, gleichzeitig aber auch ambivalente Gefühle oder gar Angst vor den Sitzungen und Deutungen haben kann. Der Patient gibt seine Einwilligung für eine Analyse, aber weder er noch die Analytikerin können im vornherein wissen, zu was er da eigentlich Ja gesagt hat, und diese Einwilligung, dieser Consent, kann in der Analyse jahrelang Thema bleiben. Zugleich ist die Analytikerin ja auch gewohnt, die geäusserten Bedürfnisse des Analysanden nicht für bare Münze zu nehmen, also zu unterscheiden zwischen expliziten Ansprüchen und eigentlichen Wünschen. Das heisst, wir müssten hier heute ziemlich viel know-how zum Thema Consent versammelt haben, und freuen uns daher auf die Diskussion. 


\section{Literarurverzeichnis}

BBC (24.05.2018). Sweden approves new law recognising sex without consent as rape, https://www.bbc.com/news/world-europe-44230786 [17.12.2018]

Benjamin, J. (1990). Die Fesseln der Liebe. Frankfurt am Main: Stroemfeld/Roter Stern.

Butler, J. (2011). Sexual Consent: Some Thoughts on Psychoanalysis and Law. Columbia Journal of Gender and Law 21 (2).

Erdheim, M. (1988). Psychoanalyse und Unbewusstheit in der Kultur. Frankfurt am Main: Suhrkamp.

Erdheim, M. (1991). Zur Lektüre von Freuds Totem und Tabu. In: Freud, S. (1991): Totem und Tabu. Frankfurt am Main: Fischer.

Ernaux, A. (2018). Erinnerung eines Mädchens. Berlin: Suhrkamp.

Laplanche, J. \& Pontalis, J.-B. (1972). Das Vokabular der Psychoanalyse. Frankfurt am Main: Suhrkamp.

Morgenthaler, F. (1994). Homosexualität, Heterosexualität, Perversion. Frankfurt am Main: Campus.

Srinivasan, A. (04.10.2018): Sex ist kein Sandwich. WOZ, https://www.woz.ch/-90f1 [19.12.2018]

Žižek, S. (2010). Die Tücke des Subjekts. Frankfurt am Main: Suhrkamp.

Žižek, S. (25.03.2017). Das Leben ist nun einmal krass. NZZ, https://www.nzz. ch/feuilleton/sex-verbote-das-leben-ist-nun-einmal-krass-ld.153338 [19.12.2018]

\section{Angaben zur Autorin und zum Autor}

Tamara Lewin, lic. phil., 1985, ist Teilnehmerin am Psychoanalytischen Seminar Zürich und arbeitet in einer psychiatrischen Tagesklinik.

Fabian Ludwig, Dr. med., 1986, ist Teilnehmer am Psychoanalytischen Seminar Zürich und arbeitet als Assistenzarzt in einem Ambulatorium. 